knowledge without investigating it, is unscientific. I noted that anthropologists and historians make analyses of shifts of power in Pacific societies while ignoring the astute and sardonic running commentary on political affairs made by Māori and Polynesian composers in their dance-songs. The analysis of social polity in Polynesian song-texts challenges anthropological explanation, but the challenge goes unanswered.

I gave an example of a way in which bicultural science can immediately be got underway. A shift towards bicultural research will come when priorities set by Mãori capture scientific effort.

During fieldwork in Northland, I concluded that Māori are hindered by lack of confidence in themselves and in their culture. Establishing confidence in Māori knowledge is a priority in bicultural science.

Sailing gave me skills of fieldwork appropriate amongst descendants of the Austronesian seafarers : to think on my feet, to meet challenges to the work; sailing gave me knowledge of one set of metaphors through which Austronesian people structure human relationships and communicate scientific ideas.

From a Stout Centre seminar on 30 May 1990

\title{
Trying to teach dynamics in a reasonable way: a suggested method for schools and universitties
}

\section{NORMAN BARBER \\ Professor Emeritus of Physics, Victoria University of Wellington}

Isaac Newton understood why planets circled around the sun instead of travelling away in straight lines. They were all falling towards the sun just as smaller objects near earth fall towards the earth. Newton could not explain how the sun could exert, an influence across great lengths of empty space, but he claimed that the strength and direction of the unknown influence (which he called a 'force') should be judged by the 'amount of motion' that it created. Unfortunately he never explained his reasons for this opinion. We are left to guess.

I urge that 'amount of motion' should be an early study of dynamics. It can be taught by using a collection of trolleys running on almost frictionless wheels. The amount of substance in each should be adjusted till any two trolleys starting at rest recoil at equal speeds when a spring is released between them. Then we know that they have equal inertia (equal mass). Perhaps each one might amount to one kilogram. The trolleys need not be alike in design or materials, but just in mass.

When the spring is released between stationary trolleys, a single one and a 'double' one (two tied together), the double one recoils less quickly. Its speed proves to be just half the speed of the single one. Yet one may claim that the 'amount of motion' is the same on both sides of the spring, arguing that two kilograms recoiling at half speed possess as much motion as one kilogram recoiling at full speed. One can try other combinations of trolleys and find that the spring always generates equal (and opposite) amounts of motion on its two sides.

The short English name for 'amount of motion' is 'momentum'. The momentum of a moving object is its velocity multiplied by the number of kilograms moving at that velocity. When objects collide and recoil it is as if some spring had briefly been trapped between the two. So momentum is gained by one object and the other gains an equal but opposite amount. The result is that the total amount of momentum has not changed. This rule is called the 'conservation of momentum'. Momentum like velocity is a vector quantity, and the rule holds no matter in what directions the different objects are moving. Many situations in dynamics can be discussed through this rule. It might be called the first rule of dynamics. Newton was 
aware of it but it seems to have been noticed first by Christiaan Huygens in Holland, who was twelve years older than Isaac Newton.

Returning to Newton's statement about 'force', the unknown influence that causes falling can be judged only by the effects that we say it produces. One effect is 'weight'. A balance shows us that the influence of the earth on two kilograms is just twice as great as its influence on one kilogram. Another effect of the influence is the gain in motion of an object that is free to fall. We might therefore study the falling motion of objects having masses of one kilogram and of two kilograms. We would watch for a gain that is doubled in the case of two kilograms, arguing that when the influence becomes doubled the effect chosen to measure it should become doubled too.

We have chosen objects heavy enough to make air friction negligible. Falling is rapid, but we can take a 'flash photograph', using perhaps ten flashes a second as a white ball falls in a darkened room before a stationary camera film. If the mass is one kilogram the distances the ball falls in successive tenths of a second are equal in metres to about $0.05,0.15,0.25,0.35$, 0.45 , and so on. The increasing speed shows that the influence of the earth causes a gain in velocity of about one metre a second in each tenth of a second.

When we do this again using a two kilogram ball, we look for a doubled effect. We may be surprised to find that the distances travelled are just the same as before. This need not worry us. To see a doubled effect we should look not at the gain in velocity but at the gain in momentum. To find momentum gain we multiply the gain in velocity by the mass of the moving object, one kilogram or two kilograms. Then clearly, the gains of the two kilogram ball are twice as great as the gains of the one kilogram ball. This shows that we should pick on the gains in momentum in order to measure the influence (the 'force'), just as Newton said. We might guess that he used the 'doubling' argument, just as we have done.

The foregoing account offers sutdents a means of demonstrating for themselves the meaning of 'momentum' and 'force'. If it were adopted as a teaching method in schools and universities I think that many students would understand dynamics better than they do now.

From a Stout Centre seminar on 6 June 1990.

\section{Father rite/right/write : lesbians, the media and the child sexual abuse controversy.}

\section{CHRIS ATMORE}

\section{$A P h D$ scholar and tutor in the Sociology Department of Victoria University of Wellington}

The paper was taken from my $\mathrm{Ph} . \mathrm{D}$ work in progress on print media constructions of lesbians in Aotearoa, from 1980 onwards. A post-structuralist reading was combined with lesbian feminist analysis in order to draw out the connections between the three homonyms: rite, right and write, and constructions of lesbians as they appear in dominant media discourses on child sexual abuse. I focussed particularly on two media topics: the use of child sexual abuse statistics in the 1988 Telethon campaign, and the Spence case, as constructed by North and

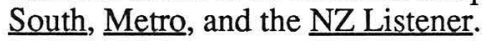

A number of typifications were present in the various media accounts, which I divided into dualisms and maskings. For example, what the Telethon and Spence accounts presented as a general wrong was the failure of researchers and child sexual abuse workers to operate within a specific scientific paradigm. Concepts like 'science', 'the facts', 'objectivity' and 'neutrality' were valued positively, and counterposed to their negatively valued opposities like 'subjectivity' and 'involvement'. The negative terms were associated with lesbian child sexual abuse workers. The location and unavoidable subjectivity of everyone in the field was denied, with lesbian feminism being presented as a conspiracy. 